\title{
BMCP1, a Novel Mitochondrial Carrier with High Expression in the Central Nervous System of Humans and Rodents, and Respiration Uncoupling Activity in Recombinant Yeast*
}

(Received for publication, August 25, 1998, and in revised form, October 2, 1998)

\begin{abstract}
Daniel Sanchis $\$$, Christophe Fleury $\ddagger$, Nathalie Chomikiๆ, Marc Goubern\|, Quinling Huang**, Maria Neverovaף, Francine Grégoireף, Juliet Easlickn, Serge Raimbaultł,

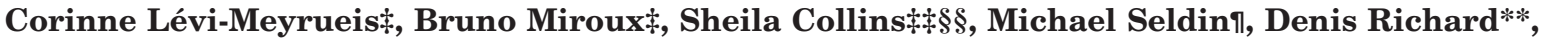

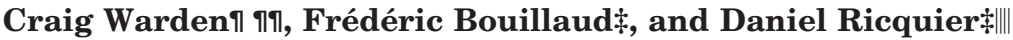

From the $\ddagger$ Centre National de la Recherche Scientifique, Centre de Recherche sur l'Endocrinologie Moléculaire et le Développement, 9 rue Jules Hetzel 92190 Meudon, France, the $\mid$ Rowe Genetics and Pediatrics, University of California, Davis, California 95616, the \|Ecole Pratique des Hautes Etudes / Institut National de la Recherche Agronomique, 78352 Jouy-en-Josas, France, the **Département de Physiologie, Faculté de Médecine, Université Laval, Québec G1K 7P4, Canada, and the \$Psychiatry and Behavioral Sciences, and Pharmacology, Duke University Medical Center, Durham, North Carolina 27710
\end{abstract}

\begin{abstract}
We report here the cloning and functional analysis of a novel homologue of the mitochondrial carriers predominantly expressed in the central nervous system and referred to as BMCP1 (brain mitochondrial carrier protein-1). The predicted amino acid sequence of this novel mitochondrial carrier indicates a level of identity of 39 , 31 , or $30 \%$, toward the mitochondrial oxoglutarate carrier, phosphate carrier, or adenine nucleotide translocator, respectively, and a level of identity of 34,38 , or $39 \%$ with the mitochondrial uncoupling proteins UCP1, UCP2, or UCP3, respectively. Northern analysis of mouse, rat, or human tissues demonstrated that mRNA of this novel gene is mainly expressed in brain, although it is 10-30-fold less expressed in other tissues. In situ hybridization analysis of brain showed it is particularly abundant in cortex, hippocampus, thalamus, amygdala, and hypothalamus. Chromosomal mapping indicates that BMCP1 is located on chromosome $X$ of mice and at Xq24 in man. Expression of the protein in yeast strongly impaired growth rate. Analysis of respiration of total recombinant yeast or yeast spheroplasts and in particular of the relationship between respiratory rate and membrane potential of yeast spheroplasts revealed a marked uncoupling activity of respiration, suggesting that although BMCP1 sequence is more distant from the uncoupling proteins (UCPs), this protein could be a fourth member of the UCP family.
\end{abstract}

Thermogenic brown fat mitochondria are characterized by a

* This research was supported by the Center National de la Recherche Scientifique (to D. Ricquier), l'Association de Recherches sur le Cancer (to F. Bouillaud), l'Institut de Recherche Servier (to D. Ricquier), and Human Frontier Science Organization contract RG 0307/ 1998-M (to D. Ricquier and D. Richard). The costs of publication of this article were defrayed in part by the payment of page charges. This article must therefore be hereby marked "advertisement" in accordance with 18 U.S.C. Section 1734 solely to indicate this fact.

$\S$ Supported by a grant from the European Union (Human Mobility Program).

$\S \S$ Supported by National Institutes of Health Grant R01 DK54024.

ๆๆ Supported by National Institutes of Health Grants NIH R01 DK52581, R01 DK 53883, and P01 HL55798-01.

||| To whom correspondence should be addressed: CNRS-CEREMOD, 9 rue Jules Hetzel 92190 Meudon, France. Tel.: 33-1-45-07-50-68; Fax: 33-1-45-07-58-90; E-mail: ricquier@infobiogen.fr. mitochondrial uncoupling protein $(\mathrm{UCP})^{1}(1,2)$, recently renamed UCP1 (3). UCP1 is uniquely found in brown adipose tissue and mutant mice null for the UCP1 gene are unable to maintain a normal body temperature in a cold environment (4). Two other members of the uncoupling protein family, UCP2 and UCP3, were recently identified $(3,5-8)$. UCP2 mRNA is widely expressed in animal or human tissues $(3,5-8)$, whereas UCP3 mRNA is largely found in skeletal muscle (6-8). These novel UCPs may explain proton leaks measured in mitochondria from most tissues and contribute to the partial coupling of respiration to $\mathrm{ADP}$ phosphorylation that exists in mitochondria from most tissues (9). Previous Northern analysis of rodent brain RNA using UCP1 cDNA or UCP2 cDNA suggested the existence of another mRNA related to the family of uncoupling proteins. ${ }^{2}$ Here, we report the cloning and functional characterization of BMCP1, a novel member of the mitochondrial carrier family that appears to be expressed in brain and, to a lesser extent, in other tissues. Although the predicted sequence of this protein is less homologous to UCP1, UCP2, or UCP3, the functional analysis of the protein when expressed in yeast indicated its potential uncoupling activity and suggested that it could be a fourth member of the UCP family.

\section{EXPERIMENTAL PROCEDURES}

Materials_Enzymes were purchased from Appligene (Illkirsch, France) and New England Biolabs (Ozyme, Montigny-le-Bretonneux, France). $\left[\alpha-{ }^{32} \mathrm{P}\right]$ dATP was obtained from Amersham Pharmacia Biotech (Les Ulis, France) and from NEN Life Science Products. Purified oligonucleotides were purchased from Eurogentec (Seraing, Belgium).

Cloning of Human and Mouse BMCP1 $c D N A-$ For the cloning of mouse BMCP1, a mouse brain cDNA library (catalog number ML5004t, CLONTECH) was screened with ${ }^{32}$ P-labeled UCP1 and UCP2 cDNAs using standardized procedures. The selection of clones was confirmed using ${ }^{32} \mathrm{P}$-labeled insert from IMAGE clone 381344 obtained from the IMAGE Consortium and originating from human retina $\left(\mathrm{GenBank}^{\mathrm{TM}}\right.$ accession number AA057007). A search in Unigene ${ }^{3}$ for uncoupling protein produced a set of expressed sequence tags that were annotated as similar to uncoupling proteins. Contig construction with the aid of further sequencing provided the information for primer design and cloning of human BMCP1. The IMAGE clone 381344 was the seed for

${ }^{1}$ The abbreviations used are: UCP, uncoupling protein; FCCP, carbonyl-cyanide $p$-trifluoromethoxyphenylhydrazone uncoupler; $\mathrm{TPP}^{+}$, tetraphenylphosphonium; contig, group of overlapping clones; PCR, polymerase chain reaction.

${ }^{2}$ D. Sanchis, C. Fleury, F. Bouillaud, and D. Ricquier, unpublished data.

${ }^{3}$ The World Wide Web address is http:/www.ncbi. 
FIG. 1. Sequence of mouse and human BMCP1. $m B M C P 1$, mouse BMCP1 predicted amino acid sequence (GenBank $^{\mathrm{TM}}$ accession number AF 076981); hBMCP1, human BMCP1 predicted sequence (GenBank $^{\mathrm{TM}}$ accession number AF078544). In the case of human BMCP1, only the residues differing from the mouse BMCP1 sequence are indicated. The three boxes identify the mitochondrial energy transfer proteins (Prosite data bank accession number PS00215).

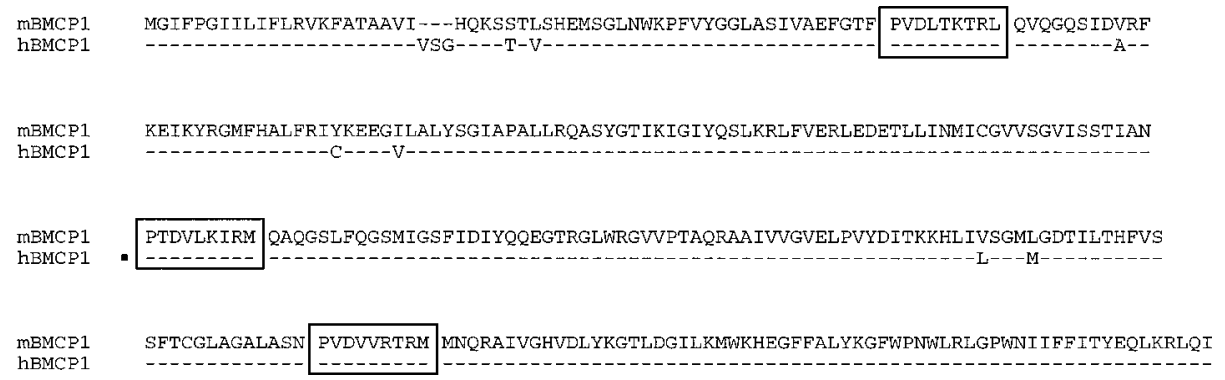

creating a contig. A $5^{\prime}$ rapid amplification of cDNA ends on human brain using the Marathon cDNA Amplification kit (CLONTECH) and primers: sense $5^{\prime}$-TGTCACCCATCAGTATCCATC- ${ }^{\prime}$ and antisense $5^{\prime}$ CAGTGGGGTGGTTCAAAG-3' produced clones containing most of the human BMCP1 coding sequence. The PCR conditions used were: $94^{\circ} \mathrm{C}$ $1 \mathrm{~min}$ and 30 cycles of $94^{\circ} \mathrm{C}$ for $30 \mathrm{~s}$ and $68^{\circ} \mathrm{C}$ for $2 \mathrm{~min}$. After characterization and complete sequencing of rapid amplification of cDNA ends products, the full-length cDNA was generated using the Advantage high fidelity PCR kit, the human brain Marathon-Ready cDNA library (both from CLONTECH), and the following PCR primer pair: sense 5'-TGGTCTCTCTCTCCCCTCAG-3' and antisense 5'CCACCCCACTGTTCTTCC-3'. The PCR conditions used were: $94{ }^{\circ} \mathrm{C}$ for $1 \mathrm{~min}$ and 30 cycles of $55{ }^{\circ} \mathrm{C}$ for $30 \mathrm{~s}$ and $72{ }^{\circ} \mathrm{C}$ for $3 \mathrm{~min}$.

Northern Blot Assays-Mouse (4-month-old B6D2 fed female) and rat (8-week-old lean Zucker fed male) multiple tissue blots were made from RNA prepared in the laboratory from different tissues using the guanidium thiocyanate procedure (10). A membrane containing $2 \mu \mathrm{g}$ of mRNA from human tissues was purchased from CLONTECH. Hybridization and washing procedures were conducted as described previously (3).

In Situ Hybridization Analysis-Three mice were intracardially perfused with paraformaldehyde. The brains were removed and cut using a sliding microtome. $25-\mu \mathrm{m}$-thick sections were collected and mounted onto poly-L-lysine coated slides. The brain sections were then hybridized as described previously (11) with mouse BMCP1 sense and antisense cRNA probes. These BMCP1 cRNA probes were generated from the 979-base pair $E c o$ RI/SacI fragment of a mouse BMCP1 cDNA subcloned into a pSELECT vector (Promega, Inc. Lyon, France), which was linearized with $B a m \mathrm{HI}$ and EcoRI for sense and antisense probes, respectively.

Assay of BMCP1 Activity in Yeast-Yeast transfections and functional analysis using flow cytometry were carried out as described previously $(3,12)$. Briefly, the diploid yeast (Saccharomyces cerevisiae) strain W303 was used for expression of BMCP1 or oxoglutarate carrier in the yeast expression vector $\mathrm{pYeDP}$. Transformants were selected for uracil auxotrophy. Yeast strains were plated on minimal medium with glucose, supplemented with adenin and tryptophan. Liquid cultures were grown in lactate minimum medium as described (13). Expression of mitochondrial carriers under the control of the gal-cyc promoter was induced by galactose in absence of glucose. Measurement of the mitochondrial membrane potential of yeast was estimated by flow cytometry in the presence of the DiOC(6)3 fluorescent probe. The preparation and use of permeabilized yeast spheroplasts was made according to Averet et al. (14). Measurement of respiration (in the presence of $6 \mathrm{~mm} \mathrm{NADH}$ ) was made with an oxygen electrode; measurement of mitochondrial membrane potential was determined with a tetraphenylphosphonium $\left(\mathrm{TPP}^{+}\right)$electrode as described elsewhere (15) under conditions where the $\Delta \mathrm{pH}$ component was low and did not vary. To compare different spheroplast preparation, the experiments were performed using the same amount of respiratory chains as determined by the maximal respiratory rate in presence of $1 \mu \mathrm{M} \mathrm{FCCP} \mathrm{uncoupler.} \mathrm{The} \mathrm{initial} \mathrm{state}$ 4 of respiration was obtained in presence of oligomycin at $10 \mu \mathrm{g} / \mathrm{ml}$. Then respiratory chain was titrated by the addition of potassium cyanide (concentrations ranging from 7 to $400 \mu \mathrm{M}$ ). Under these conditions, the uptake of $\mathrm{TPP}^{+}$is proportional to the membrane potential, and independent preparations of spheroplasts or mitochondria can be accurately compared.

\section{RESULTS AND DISCUSSION}

Cloning of BMCP1 cDNA, Sequence of Predicted BMCP1 Protein in Mouse and Man, and Comparison with Other UCPs and Other Mitochondrial Carriers-A mouse brain cDNA library was screened using mouse UCP1 and mouse UCP2 cDNA as probes. A number of candidates clones were further screened using the human expressed sequence tag IMAGE clone 381344 (see "Experimental Procedures"). This expressed sequence tag was similar but not identical to hUCP1, hUCP2, or hUCP3. The screening led to the cloning of a mouse cDNA referred to here as BMCP1 (brain mitochondrial carrier protein- $\underline{1}$ ). The fulllength cDNA of human BMCP1 was generated as described under "Experimental Procedures." The predicted amino acid sequence of mouse and human BMCP1 is given in Fig. 1. Mouse and human BMCP1 are highly similar and share a level of identity of $97 \%$. The predicted sequence of the mouse protein is 322 amino acids (calculated molecular mass, 36,019 daltons), and the predicted sequence for the human protein is 325 residues. The BMCP1 sequence shows the three motifs characteristic for the triplicate mitochondrial carriers (boxed in Fig. 1) and also predicts the existence of six transmembrane domains (not shown). Differences between the human and mouse sequences at amino acids 23-25 (VSG) are probably due to sequence polymorphisms, because we also identified human BMCP1 cDNA, which lacks the corresponding 9-base pair VSG motif. The levels of identity between BMCP1 and UCP1, UCP2, and UCP3 are 34, 38, and 39\%, respectively. Therefore, the predicted BMCP1 sequence is less homologous to the other animal UCPs, which share 60-70\% identity. BMCP1 sequence is $38 \%$ identical to the plant Solanum tuberosum UCP sequence (16). A difference between BMCP1 predicted sequence and the other UCPs is in the $20 \mathrm{~N}$-terminal amino acid residues in BMCP1 that are absent in UCP1, UCP2, and UCP3. This $\mathrm{N}$-terminal sequence is significantly distant from known motifs implicated in the targeting of mitochondrial precursors to mitochondria. Whether this N-terminal part of BMCP1 is involved in targeting of BMCP1 into mitochondria is under investigation; preliminary data indicate that in vitro translated BMCP1 is incorporated in isolated mitochondria. ${ }^{4}$ In other respects, BMCP1 is as homologous to the mitochondrial oxoglutarate carrier as it is to the other UCPs (39\% identity, alignment not shown) and shares $30 \%$ identity with the adenine nucleotide translocator 1 .

Tissue Distribution of BMCP1 Transcripts in Rodents and Man-Northern blot analysis of BMCP1 mRNA expression in mouse, rat, or human tissues revealed that BMCP1 is highly expressed in brain as a 1.8-kilobase mRNA (Fig. 2). In addition to the primary expression in brain, BMCP1 mRNA was also detected to a lesser in heart, skeletal muscle, gut, lung, kidney, uterus, testis, and adipose tissues of mice, whereas no expression was measured in liver of mice. However, relative to the brain, the levels of expression of BMCP1 mRNA in other tissues of mice (quantitated using a PhosphorImager) were 13fold less in heart, 28-fold less in muscle, 9-fold less in gut, 7.5-fold-less in lung, 31-fold less in spleen, 7-fold less in kidney, 10 -fold less in uterus, 7 -fold less in testis, 9-fold less in periovarian fat, and 30-fold less in brown adipose tissue. A weak

\footnotetext{
${ }^{4}$ D. Sanchis and K. Prip-Buus, unpublished data.
} 

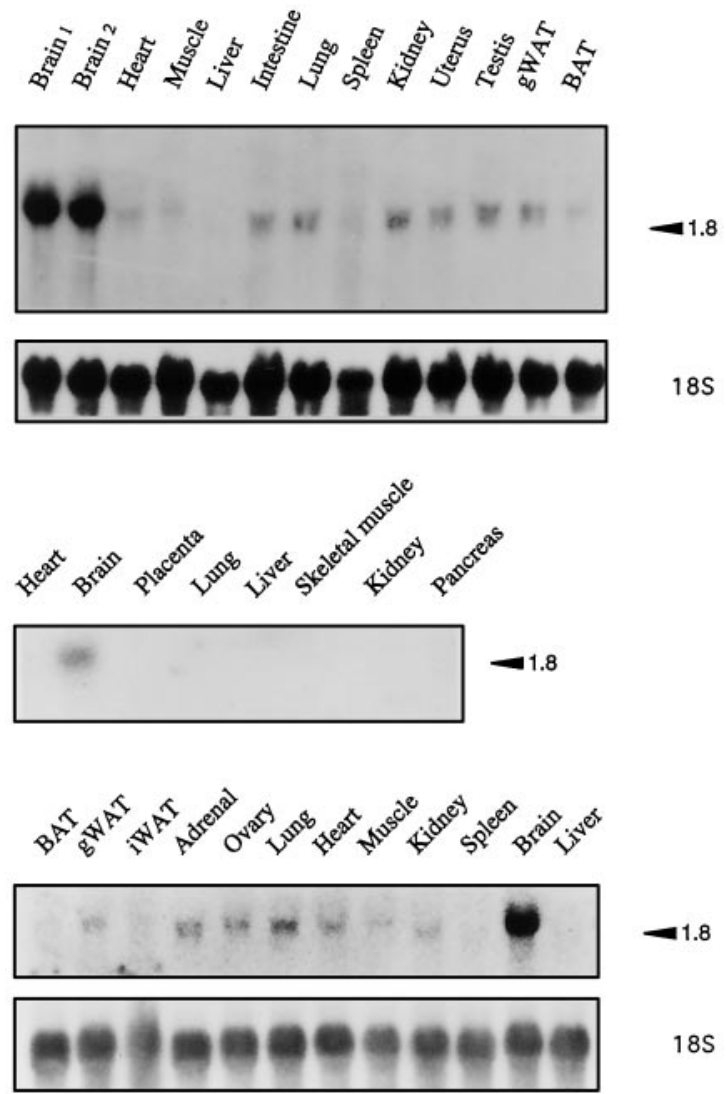

FIG. 2. Tissue distribution of BMCP1 mRNA in rodent or human tissues. Top panel, total RNA $(20 \mu \mathrm{g})$ from various mouse tissues was analyzed for the expression of BMCP1 mRNAs using ${ }^{32} \mathrm{P}$-labeled BMCP1 mouse cDNA. Brain 1 and Brain 2 correspond to preparation of RNA made from different animals. BAT, brown adipose tissue; $g W A T$, gonadal (periovarian) white adipose tissue. Middle panel, $\operatorname{poly}(\mathrm{A})^{+} \mathrm{RNA}$ $(2 \mu \mathrm{g})$ from various human tissues was analyzed for the expression of BMCP1. Bottom panel, total RNA $(20 \mu \mathrm{g})$ from various rat tissues was analyzed for the expression of BMCP1 mRNA. gWAT, gonadal white adipose tissue; $i W A T$, inguinal white adipose tissue. The size of BMCP1 mRNA (in kilobases) is indicated on the right-hand side of the figure. Staining of the membranes with methylene blue (not shown) and hybridization of an $18 \mathrm{~S}$ RNA probe were used to check that an identical amount of mouse or rat RNA was loaded in every lane.

expression of BMCP1 mRNA was observed in lung, adrenals, gonadal fat, ovary, heart, and kidney of rats. In human tissues, a clear signal was obtained in brain using Northern analysis. Some expression was also noticed in human testis and pituitary using a dot blot containing RNA from 50 different human tissues and purchased from CLONTECH (data not shown).

In Situ Hybridization Analysis of BMCP1 mRNA in BrainFig. 3 demonstrates the distribution of BMCP1 mRNA at the level of the tuberal region of the mouse hypothalamus. The expression was particularly abundant in the cortex, the hippocampus, the thalamus, the amygdala, and the hypothalamus. This distribution substantially differs from that of UCP2 mRNA (11) in that it is more ubiquitous. The exact location of cells expressing BMCP1 mRNA remains to be delineated.

Effect of BMCP1 on Growth Rate of Yeast-To examine the activity of BMCP1 in mitochondria and test for possible functional uncoupling activity, BMCP1 protein was expressed in yeast as previously reported for UCP1 $(12,13)$ or UCP2 (3). Upon induction of BMCP1 expression, the growth rate of yeast was significantly reduced relative to the vector control, whereas induction of expression of the mitochondrial oxoglutarate carrier had no effect (Fig. 4). BMCP1 expression increased the instantaneous generation time measured in liquid culture (241 $\pm 3 \min , n=5)$ when compared with yeast ex-

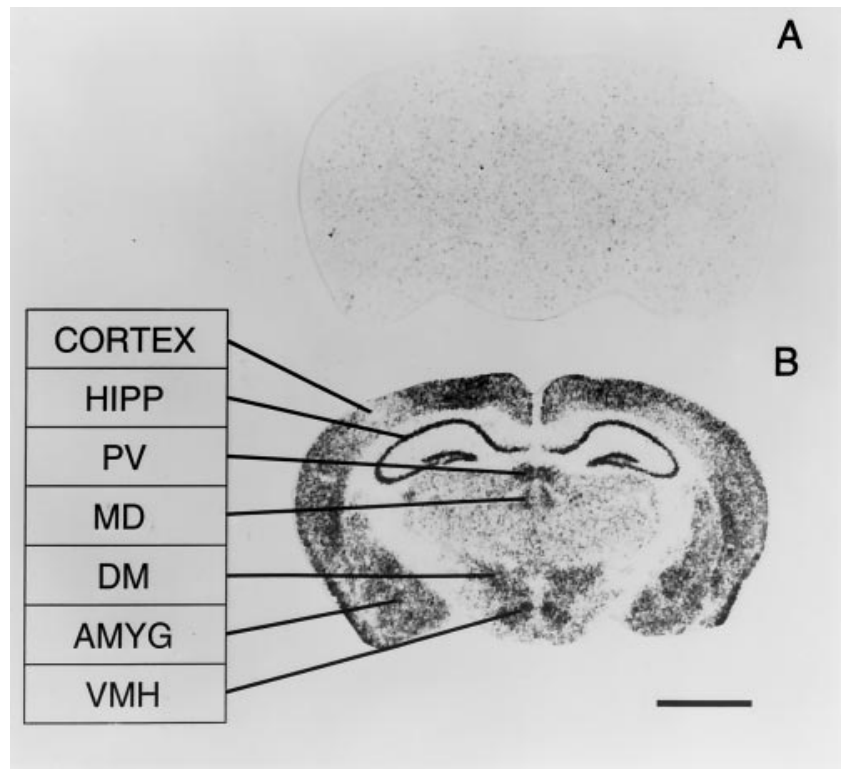

FIG. 3. In situ hybridization analysis of BMCP1 mRNA in brain. Film autoradiograms of coronal brain sections hybridized with radiolabeled sense $(A)$ and antisense $(B)$ riboprobes. The sections were taken at the level of the ventromedial hypothalamic nucleus. At this level, the intensity of the hybridization signal was strong and visible in many regions. The scale bar corresponds to $2.0 \mathrm{~mm}$. Amyg, amygdala; $D M$, dorsomedial hypothalamic nucleus; Hipp, hippocampus; $P V$, paraventricular thalamic nucleus; $M D$, mediodorsal thalamic nucleus; $V M H$, ventromedial hypothalamus.

pressing no recombinant protein $(185 \pm 2 \mathrm{~min}, n=5, p<$ 0.001 , Student's $t$ test). A similar inhibition of growth was observed in yeast overexpressing UCP1 or UCP2 $(3,12,13)$.

Effect of BMCP1 on Mitochondrial Coupling in Yeast-To measure the effect of BMCP1 on the mitochondrial membrane potential, we used flow cytometry analysis of intact yeast labeled with the fluorescent dye $\operatorname{DiOC(6)3~(Fig.~5)~and~also~ana-~}$ lyzed permeabilized yeast spheroplasts (Fig. 6). Induction of expression of BMCP1 for $5 \mathrm{~h}$ lead to a marked decrease of the staining with the mitochondrial pontential-sensitive probe of whole yeast analyzed by flow cytometry as previously observed with UCP1 (12), suggesting an uncoupling activity of respiration. In contrast, expression of the oxoglutarate carrier in yeast did not alter staining of the mitochondria. However, a decreased staining of mitochondria by the fluorescent probe does not necessarily reflect a decreased coupling of respiration because a reduction of the number of mitochondria or an increase of some other protonmotive force consumers would have provoked a similar effect. In fact, full demonstration of uncoupling activity of a mitochondrial protein requires additional analysis of mitochondrial bioenergetics. The method allowing analysis of an imperfect coupling was first reported by Nicholls (17) and modified by Brand et al. (18). We have investigated the bioenergetics parameters concerning uncoupling effect of BMCP1 according to this method. For this purpose, nystatin permeabilized yeast spheroplasts are an accurate material easier to prepare than mitochondria (14).

Fig. $6 A$ shows the simultaneous recording of respiratory rate and mitochondrial membrane potential $\left(\mathrm{TPP}^{+}\right.$uptake) of control or BMCP1 spheroplasts. Addition of $6 \mathrm{~mm} \mathrm{NADH}$ to permeabilized spheroplasts led to a true state 4 respiration (checked to be oligomycin-insensitive), which was enhanced in BMCP1 spheroplasts concomitantly with a lower membrane potential. Addition of $2 \mathrm{~mm} \mathrm{ADP}$ promoting state 3 respiration decreased membrane potential to the same level and revealed a poor coupling of respiration of BMCP1 mitochondria compared with control mitochondria. The two traces on the right-hand 
pYeDP

FIG. 4. Functional activity of mouse BMCP1 expressed in yeasts: effects on growth. Photographs of yeast colonies growing on agar plates in minimum medium $40 \mathrm{~h}$ in presence of glucose (GLU., inhibition of expression vector) or galactose $(G A L$., activation of expression vector) are shown. The yeasts were either transformed by the empty pYeDP expression vector or the same vector containing either the mouse oxoglutarate carrier cDNA $(O G C)$ or the mouse BMCP1 cDNA.

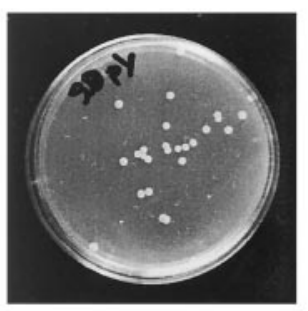

GLU.
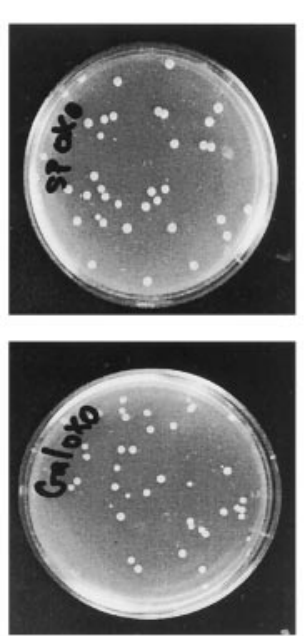

BMCPI
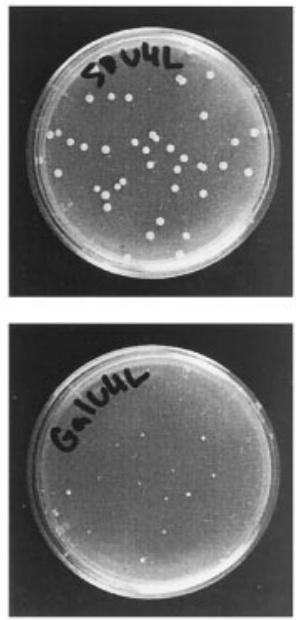

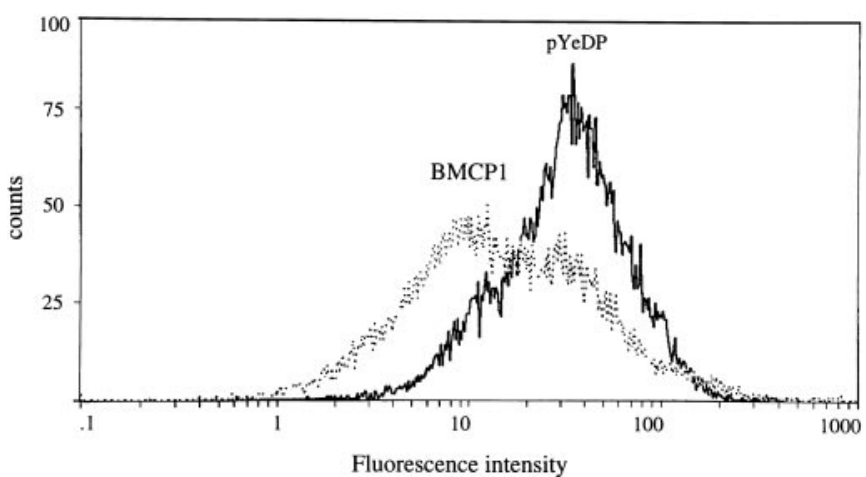

FIG. 5. Functional activity of mouse BMCP1 expressed in yeasts: flow cytometry analysis of mitochondrial membrane potential using whole yeasts. Flow cytometry analysis of mitochondrial membrane potential following the expression of BMCP1 in S. cerevisiae; comparison with empty vector or vector expressing the mitochondrial oxoglutarate carrier. The number of yeast is represented on the $y$ axis. The $x$ axis is a logarithmic scale of fluorescence intensity. This schema is representative of four independent experiments. The curves correspond to yeast transfected either with an empty vector ( $\mathrm{pYeDP}$, continuous line) or an expression vector for mouse BMCP1 (BMCP1, dotted line) and cultured for $5 \mathrm{~h}$ in presence of galactose (protein expression inducer). Yeast expressing the mitochondrial oxoglutarate carrier showed a pattern totally identical to control yeast.

side of Fig. $6 A$ show that addition of FCCP stimulated 3.1-fold the respiratory activity of control spheroplasts, whereas it stimulated only 1.8-fold the respiration of BMCP1 spheroplasts. This result was consistent with several experiments where we used different preparations of spheroplasts corresponding to independent cultures of yeasts, as well as independent transformations of yeast to rule out the eventuality of a bias due to the recipient cells. The respiratory control ratio values (defined as the ratio between respiratory rate in presence of FCCP, and respiratory rate in state 4 of respiration) of spheroplasts isolated from yeast overexpressing BMCP1 or not overexpressing BMCP1 were $2.02 \pm 0.08(n=17)$ and $3.73 \pm$ $0.22(n=26)$, respectively (mean \pm S.E., $p<0.001$, Student's $t$ test). These data confirmed a lower coupling of respiration in yeast expressing BMCP1 compared with control yeast.

Nicholls (17) and Brand et al. (18) have shown that the measurement of the force/flux relationship between mitochondrial membrane potential and state 4 respiratory rate (in presence of oligomycin, i.e. in nonphosphorylating mitochondria) undoubtedly demonstrates and quantifies proton leaks through inner mitochondrial membrane. This method was applied to yeast spheroplasts. To obtain curves reported in Fig. 6B,
A
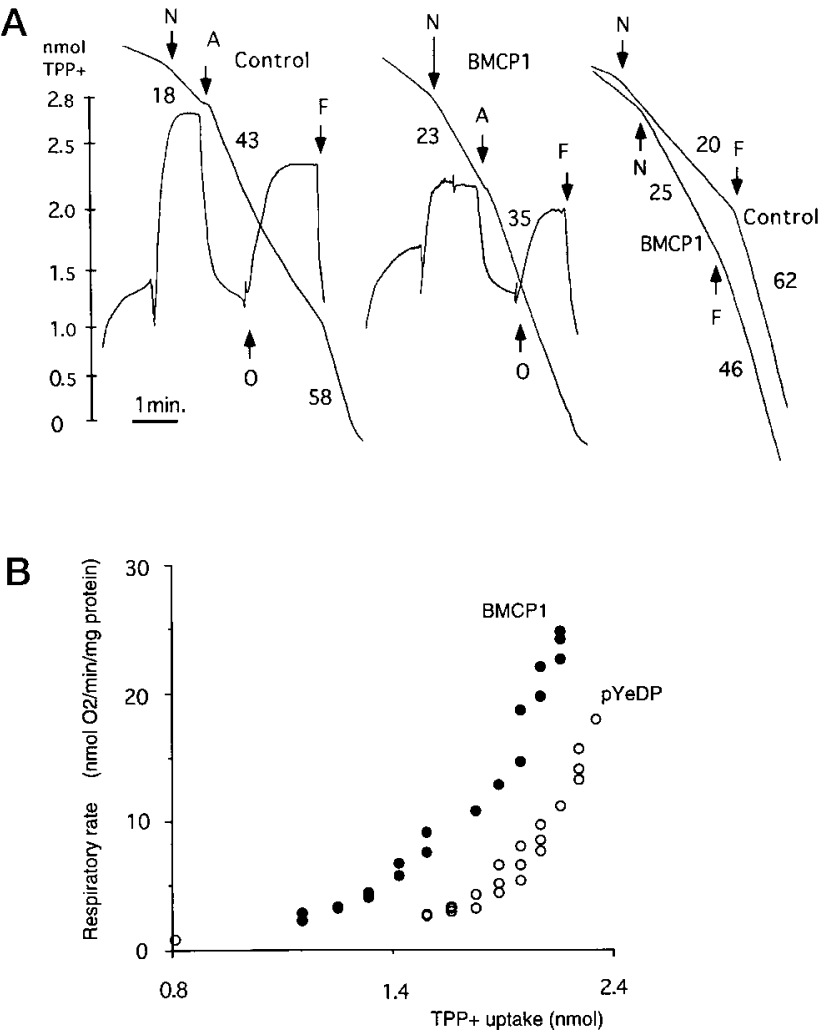

FIG. 6. Functional activity of mouse BMCP1 expressed in yeasts: analysis of respiratory rate and mitochondrial membrane potential using yeast spheroplasts. $A$, simultaneous recording of mitochondrial membrane potential (biphasic curves) and oxygen consumption (lines) of control (pYeDP plasmid) or BMCP1 yeast spheroplasts. Mitochondrial membrane potential was estimated from uptake of $\mathrm{TPP}^{+}\left(x\right.$ axis, $\mathrm{TPP}^{+}$uptake is proportional to the mitochondrial membrane; see "Experimental Procedures"). Respiratory rate was measured using an oxygen electrode (the numbers along the respiratory traces correspond to $\mathrm{nmol} 0_{2} / \mathrm{min} / \mathrm{mg}$ protein). $N$, NADH ( $6 \mathrm{~mm}$, respiratory substrate); $A, \operatorname{ADP}(2 \mathrm{~mm}) ; O$, oligomycin $(10 \mu \mathrm{g} / \mathrm{ml})) ; F, \operatorname{FCCP}(7$ $\mu \mathrm{M})$. The two traces on the right-hand side of the figure show the respiratory traces of control or BMCP1 spheroplasts used to perform the experiment shown in panel $B$; the exact values of respiratory control ratio corresponding to the curves shown are 3.1 and 1.78 for pYeDP and BMCP1 spheroplasts, respectively. $B$, relationship between respiratory rate $\left(\mathrm{nmol} \mathrm{O}_{2} / \mathrm{min} / \mathrm{mg}\right.$ protein) of control ( $\mathrm{pYeDP}$, open circles) or BMCP1 (BMCP1, closed circles) yeast spheroplasts, and mitochondrial membrane potential (uptake of $\mathrm{TPP}^{+}, x$ axis). This experiment was performed in the presence of oligomycin using a respective amount of control or BMCP1 spheroplast corresponding to the same amount of respiratory chains as determined from respiratory trace in presence of FCCP as shown in panel $A$. 
amounts of control and BMCP1 permeabilized spheroplasts were normalized to assume the same FCCP uncoupled maximal respiratory rate. Thus it is likely that the same quantity of mitochondria reflected by the same maximal activity of the respiratory chain was present. Data shown in Fig. $6 B$ were obtained in presence of oligomycin, and NADH oxidation was progressively slowed by potassium cyanide titration according to published protocols (Refs. 17 and 18 and see "Experimental Procedures"). As the rate of substrate oxidation decreases, the membrane potential (recorded with a $\mathrm{TPP}^{+}$electrode in conditions where $\mathrm{TPP}^{+}$uptake is proportional to membrane potential, the mean component of the protonmotive force) and the proton motive force decrease. The comparison of the force/flux curves of BMCP1 spheroplasts with control spheroplasts shows an enhanced respiratory rate regardless of the value of membrane potential. The decreased membrane potential associated with increased respiratory rate in BMCP1 spheroplasts suggested out the possibility that our observations might be due to an artifactual consequence of a lower mitochondrial content. According to Nicholls (17) and Brand et al. (18), these data demonstrate that BMCP1 expression induced an uncoupling of mitochondrial respiration in recombinant yeast and indicate that addition of BMCP1 to mitochondria increased a proton leak.

On the basis of such data, it may be proposed that BMCP1 participates to the mitochondrial proton leak measured in brain mitochondria (19). Whether BMCP1 modulates the efficiency of respiration in certain brain cells in vivo remains to be investigated. In preliminary experiments we tested the hypothesis of a regulation of BMCP1 activity in yeast by fatty acids or nucleotides that are known activators or inhibitors of UCP1 (1) and observed no effect. The possible regulation of BMCP1 activity by particular ligands will be investigated. Actually, an uncoupling activity of a mitochondrial carrier moderately homologous to the UCPs is not entirely surprising because it was proposed that the adenine translocator of mitochondria, which shares $30 \%$ identity with UCP1, UCP2, UCP3, or BMCP1, can mediate the uncoupling effect of fatty acids (20). Conversely, the data presented here do not rule out another activity of BMCP1.

Chromosomal Location-We determined the chromosomal location of BMCP1 in mice and humans. Both localizations are consistent with the presence of a single BMCP1 locus on the $\mathrm{X}$ chromosome. In humans a sequence tagged site (G23624) was derived from IMAGE clone 33681, GenBank ${ }^{\mathrm{TM}}$ accession number R44688, which is part of the human BMCP1 cluster. The transcript marker WI-13677 was mapped to human chromosome X between DXS1047 and DXS994, placing BMCP1 between 150 and 151 centimorgans on the human gene map (300.5 centiroentgens on the WI radiation hybrid map). The chromosomal location of mouse BMCP1 was determined in a well characterized $\mathrm{C} 3 \mathrm{H} \times$ Mus spretus backcross panel, as described previously (3). A restriction fragment length polymorphism was used to map mBMCP1 to the X chromosome at 12.5 centimorgans, co-incident with Gria3. This location predicts that the human homologue would be on the X chromosome at $\mathrm{Xq} 24$, which is the same location identified by sequence tagged site mapping. The mouse BMCP1 locus is included in the confidence intervals for two mouse quantitative trait loci for body weight. These quantitative trait loci were named body weights 1 and 2. BMCP1 may be a positional candidate for these body weight quantitative trait loci, although they are very broad, including most of the $\mathrm{X}$ chromosome. Interestingly, the ADP/ATP translocator-2 (ANT2) gene, which is also part of the same mitochondrial carrier family as BMCP1, has been mapped to Xq24. This suggests the possibility that BMCP1 and ANT2 may be duplicated genes with divergent functions.

In conclusion, we report here the cloning of cDNAs encoding a new mouse or human mitochondrial carrier protein that we propose to refer to as BMCP1. BMCP1 gene is located on chromosome $\mathrm{X}$. On the basis of the predicted amino acid sequence, this new protein belongs to the family of mitochondrial carriers and is equally homologous to mitochondrial oxoglutarate carrier and UCPs. However, when it is expressed in yeasts, the detailed analysis of the relationship between the respiratory rate and the mitochondrial membrane potential indicates that BMCP1 behaves as a potent uncoupler of respiration. The predominant expression of BMCP1 in brain raises the question as to the physiological significance and specific role for BMCP1 in neurons, for example the possibility to modulate ATP/ADP ratio or level of reactive oxygen species.

Acknowledgments-We acknowledge the excellent technical assistance of M. F. Chapey and Dr. G. Durand for support during these studies.

\section{REFERENCES}

1. Nicholls, D. G., and Locke R. (1984) Physiol. Rev. 64, 1-64

2. Ricquier, D Casteilla, L and Bouillaud, F (1991) FASEB J. 5, 2237-2242

3. Fleury, C., Neverova, M., Collins, S., Raimbault, S., Champigny, O., LéviMeyrueis, C., Bouillaud F., Seldin, M. F., Surwitt, R. S., Ricquier, D., and Warden, C. H. (1997) Nat. Gen. 15, 269-272

4. Enerbäck, S., Jacobsson, A., Simpson, E., Guerra, C., Yamashita, H., Harper M., and Kozak, L. P. (1997) Nature 387, 90-94

5. Gimeno, R., Dembski, M., Weng, X., Deng, N., Shyjan, A., Gimeno, C., Iris, F., Ellis, S., Woolf, E., and Tartaglia, L. (1997) Diabetes 46, 900-906

6. Boss, O., Samec, S., Paoloni, G. A., Rossier, C., Dulloo, A., Seydoux, J., Muzzin, P., and Giacobino, J. P. (1997) FEBS Lett. 408, 39-42

7. Vidal-Puig, A, Solanes, G, Grujic, D, Flier, J. S., and Lowell, B. B. (1997) Biochem. Biophys. Res. Commun. 235, 79-82

8. Gong, D.-W., He, Y., Karas, M., and Reitman, M. (1997) J. Biol. Chem. 272, $24129-24132$

9. Rolfe, D. F. S., and Brown, G. C. (1997) Physiol. Rev. 77, 731-758

10. Chomczynski, P., and Sacchi, N. (1987) Anal. Biochem. 162, 156-159

11. Richard, D., Rivest, R., Huang, Q., Bouillaud, F., Sanchis, D., Champigny, O., and Ricquier, D. (1998) J. Comp. Neurol. 397, 549-560

12. Bouillaud, F., Arechaga, I., Petit, P. X., Lévi-Meyrueis, C., Casteilla, L., Laurent, M., Rial, E., and Ricquier, D. (1994) EMBO J. 13, 1990-1997

13. Arechaga, I., Raimbault, S., Prieto, S., Lévi-Meyrueis, C., Zaragoza, P. Miroux, B., Ricquier, D., Bouillaud, F., and Rial, E. (1993) Biochem. J. 296, 693-700

14. Averet, N., Fitton, V., Bunoust, O., Rigoulet, B., and Guérin, B. (1998) Mol. Cell Biochem. 184, 67-79

15. Beauseigneur, F., Goubern, M., Chapey, M.-F., Gresti, J., Vergely, C., Tsoko, M., Demarquoy, J., Rochette, L., and Clouet, P.(1996) Biochem. J. 320, 571-576

16. Laloi, M., Klein, M., Riesmeier, J. W., Muller-Rober, B., Fleury, C, Bouillaud, F., and Ricquier, D. (1997) Nature 389, 135-136

17. Nicholls, D. G. (1974) Eur. J. Biochem. 50, 305-315

18. Brand, M. D., Chien, L.-F., Ainscow E. K., Rolfe D. F. S., and Porter, R. K. (1994) Biochim. Biophys. Acta 1187, 132-139

19. Rolfe, F. S., Hulbert, A. J., and Brand, M. (1994) Biochim. Biophys. Acta 1118, $405-416$

20. Skulachev, V. P. (1998) Biochim. Biophys. Acta 1363, 100-124 